\title{
EINIGE MODIFIKATIONEN IN DER INSTRUMENTELLEN BESAMUNG VON BIENENKÖNIGINNEN
}

\author{
Quelques modifications de l'appareil à insémination \\ des reines d'abeilles \\ H.H.W. VELTHUIS und M. J. SOMMEIJER \\ Laboratorium für vergleichende Physiologie der Universität, Utrecht (Niederlande)
}

\section{SUMMARY}

SOME MODIFICATIONS OF THE APPARATUS FOR THE INSEMINATION

OF HONEYBEE QUEENS

Some modifications have been made to the MaCKEnSEN-RoBerts apparatus. The syringeholder is not attached directly on the mountingblock $(\mathrm{H})$ but on a thin rod (G), permitting a lateral slip and more accurate adjustments. Besides, the capillary glass syringe is broken off at the superior end, and the two parts are united by a plastic tube in order to offer greater elasticity.

Für die Durchführung von Experimenten, über die wir auf dem Münchner Apimondia-Kongress ${ }^{1}$ bereits berichtet haben, benutzten wir die Technik der instrumentellen Besamung. In diesem Beitrag wollen wir einige Änderungen der allgemein üblichen Technik beschreiben, die uns die Ausführung der Besamung wesentlich erleichtert haben ${ }^{2}$.

In wesentlichen gleicht unser Apparat demjenigen von MACKENSEN-RoBerts : die Königin befindet sich gefesselt in einem Königinnenhalter (A), der in jeden beliebig schrägen Stand gestellt werden kann. Die Ventral- (B) und Dorsalhäkchen (C) sind mittels einer Klemme (D) drehbar an einer vertikalen Stativachse (E) befestigt und ermöglichen das Öffnen der Stachelkammer der Königin. Die Häkchen wurden nach VAN LAERE ${ }^{3}$ aus speziellem Stahl angefertigt.

1. SommeiJer M. J. und Velthuis H.H.W. (1969). The acceptance of queens without mandibular glands after artificial insemination. XXII. Int. Bienenzüchter-Kongress, München 1969. rates.

2. Wir danken Herrn W. MaAsse für seine Hilfe beim Entwurf und der Konstruktion des Appa-

3. Wir danken Herrn Dr. O. Van Laere für seine Einführung in die Probleme der instrumentellen Besamung. 
Die Besamungsspritze (F) ist somit an der Stativachse des dorsalen Häkchens aufgehängt. Diese Befestigung unterscheidet sich wesentlich von der bisher üblichen. Statt einer direkten Befestigung des Spritzhalters an der vertikalen Säule haben wir eine schräge indirekte Verbindung hergestellt, indem wir an dem Spritzhalter eine dünnere Stange (G) befestigten, wie aus der Abbildung ersichtlich ist. Bei der Insemination wird die Schraube, mit der die dünnere Stange an dem Befestigungsblock $(\mathrm{H})$ der vertikalen Säule verbunden ist, nur wenig angedreht, wodurch eine sehr geschmeidige laterale Bewegung der Spitzenmündung in die Stachelkammer ermöglicht wird. Da diese Schraube gleichzeitig eine horizontale Drehachse darstellt, kann auch eine Neigung der dünneren Stange und damit das Einführen der Spitzenmündung in die Scheide der Königin leicht ausgeführt werden. Dorso-ventrale Bewegungen der Spitzenmündung innerhalb der Stachelkammer sind ebenfalls möglich durch eine zweite horizontale Drehachse zwischen dünner Stange und Spritzhalter (I). Die hier vorhandene Klemmschraube wird bei der Insemination nur wenig angedreht.

Schliesslich kann die Spritze, die mittels einer federnden Klemme (K) an dem Spritzhalter befestigt ist, abwärts geschoben werden. Auch diese Klemmschraube wird nur so wenig, zugedreht, als notwendig ist, um ein Herunterrutschen der Spritze zu vermeiden. Mittels dieser sehr beweglichen Aufhängung der Spritze kann jede beliebige Bewegung ihrer Spitze innerhalb der Stachelkammer glatt und sicher ausgeführt werden.

Die Gestaltung der Spritze ist wie bei Mackensen, nur verwendeten wir statt Acrylspitzen Glasspitzen, wie auch VAN LAERE sie bevorzugt ${ }^{4}$. Unsere Glasspitzen sind aber erheblich dünner und auch schlanker als die von VaN LAERE. Sie werden angefertigt aus Glaskapillaren mit einem Aussendurchmesser von $1,6 \mathrm{~mm}$ und einem Innendurchmesser von $0,9 \mathrm{~mm}$. Diese werden über einer kleinen Gasflamme in die Länge gezogen. Wo der innere Durchmesser auf $0,18 \mathrm{~mm}$ herabgesunken ist, wird die Kapillare abgebrochen. Der äussere Durchmesser ist dann in den meisten Fällen unter $0,3 \mathrm{~mm}$ abgesunken. Wenn nötig wird die Kapillare durch Abschleifen etwas gekürzt, womit sowohl Innen- als Aussendurchmesser zunehmen. Dann wird die Mündung mit in Bienenwachs eingeschmolzenem rotem Polierpulver völlig rund und glatt poliert. Schleifen und polieren werden mittels eines Zahnartzbohrgerätes unter dem Binokular durchgeführt. Die in dieser Weise hergestellten Spitzen haben in der Regel wie die Spitze nach VESELY 5 ein fast zylinderförmiges Spitzenende. Dadurch waren wir imstande, auch kleinere Königinnen ohne viel Schwierigkeiten zu inseminieren.

Weil unsere Spitze selbstverständlich sehr zerbrechlich ist, haben wir die Kapillare in ihrem oberen Teil durchgeschnitten und die zwei Teile durch einen Plastikschlauch wieder miteinander verbunden. Nur grobes Anstossen der Kapillare lässt nun die Spitze noch zerbrechen. Ein zweiter Vorteil dieses Plastikschlauches ist, dass jeder Widerstand innerhalb der Königin zugleich bemerkbar wird, indem das Spitzenende den ausgeführten Bewegungen nicht mehr folgt.

Bei der Insemination braucht man keine Vaginalsonde. Die Spitze wird mit kleinen kreisförmigen Bewegungen in die Vagina eingeführt. Sobald man auf die Valvula vaginalis stosst, was an dem Mitbewegen der Scheidewand ersichtlich ist, wird die Spitze dorsalwärts gelenkt und kann man durch ähnliche Bewegung wie mit der Vaginalsonde die Valvula niederklappen.

4. Siehe über die Glasspitze nach Van IaErE: V. Vesely und H. Rutrner, Apparaturen zur künstlichen Besamung der Bienenköniginnen, in : Die instrumentelle Besamung der Bienenkönigin, herausgegeben in Auftrag der Apimondia von F. Ruttner, Bukarest 1969. Da findet man auch eine Beschreibung des Apparates nach Mackenser-Roberts.

5. VeSExy V. (1965). Die zylinderförmig beendete Inseminations-spitze ermöglicht die künstliche Besamung, ohne dass die Sonde gebraucht wird, und die Samenabnahme direkt von den Samenleitern der Drohnen. Ref. Symposion XX. Int. Bienenzüchter Kongress, Bukarest. Auch : Luerari Scientifice $7: 57-61$ (1966). 
Zum Ansaugen des Spermas kann man den viereckigen Balken (L), an dem Königinnenhalter und Stativsäulen befestigt sind, um seine Längsachse drehen und zugleich die Spritze dem Balken parallel stellen.
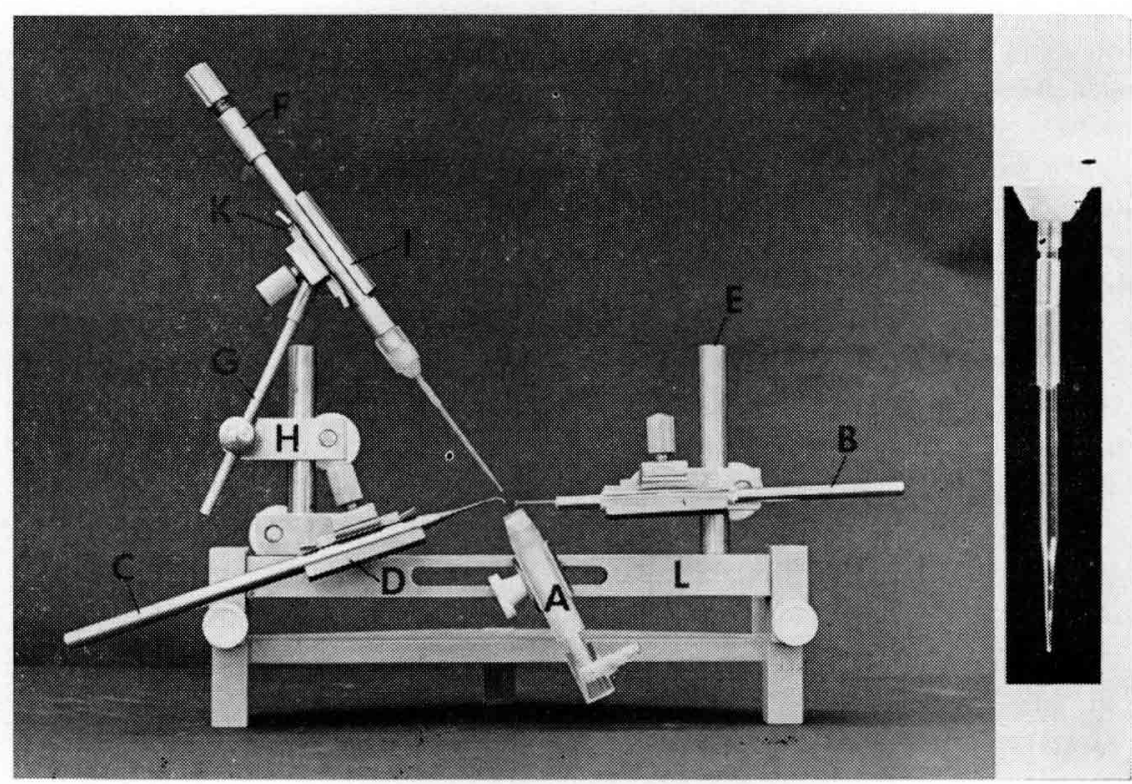

\section{Авв. 1. - Der Apparat und die Spitze}

A : Königinnenhalter, B : Ventralhäkchen, C : Dorsalhäkchen, D : Klemme der Häkchen, E : Stativachse; F : Spritze; G : Dïnnere Stange; H : Befestigungsblock derselben; I : Spritzhalter; K : Klemme der Spritze; I. : Viereckige Balke.

\section{Fig. 1. - Appareil et pointe}

A : Logement de la reine; B : Crochet ventral; C : Crochet dorsal; D : Pince du crochet; E : Axe de support; F : Ssringue; G : Tringle mince; H : Bloc de fixation de la tringle; I : Porte-seringue; $K$ : Pince de la seringue; I. : Barre rectangulaire.

Eingegangen im Mai 1970. Reçu pour publication en mai 1970.

\section{RÉSUMÉ}

Pour l'essentiel l'appareil est semblable à celui de Mackensen-Roberts. Il se différencie en ce qui concerne le mode de fixation de la seringue (F) sur l'axe de support du crochet dorsal (C). Au lieu d'une fixation directe sur la colonne porte-outil verticale, on a imaginé une liaison indirecte oblique, au moyen d'une tringle beaucoup plus mince (G). Lors de l'insémination, la vis reliant la tringle au bloc de fixation de la colonne verticale est légèrement actionnée pour obtenir un mouvement 1atéral très souple de la pointe dans la chambre de l'aiguillon. Au moyen d'une inclinaison de la tringle, on peut procéder facilement à l'introduction de la pointe dans le vagin de la reine. Il est 
également possible d'obtenir des mouvements dorsaux-ventraux de la pointe à l'intérieur de la chambre de l'aiguillon, grâce à un deuxième axe rotatif horizontal $(\mathrm{K})$, situé entre la tringle et le porte-seringue. Enfin la seringue, fixée sur le porte-seringue par une pince élastique, peut glisser vers le bas. On peut ainsi effectuer avec la pointe n'importe quel mouvement à l'intérieur de la chambre de l'aiguilion. On a utilisé des pointes en verre, beaucoup plus fines que celles de VAN LAEre. Elles sont fabriquées à partir de capillaires de verre, étirés dans la longueur à la flamme d'un brûleur à gaz et brisés à l'endroit où le diamètre intérieur est de $0,18 \mathrm{~mm}$. La pointe est ensuite arrondie et polie sous la loupe binoculaire, au moyen d'une fraise de dentiste. Les pointes ainsi façonnées ont une extrémité presque cylindrique. La pointe étant évidemment très fragile, nous avons coupé le capillaire dans sa partie supérieure et relié les deux parties à l'aide d'un conduit de plastique. Ainsi seule une forte poussée du capillaire peut briser la pointe. Par ailleurs ce système présente l'avantage de mettre en évidence toute résistance à l'intérieur de la reine, puisque, dans ce cas, l'extrémité de la pointe ne suit plus les mouvements imprimés.

Il n'est pas besoin de sonde vaginale pour l'insémination. La pointe est introduite dans le vagin au moyen de petits mouvements circulaires. Dès que l'on a heurté la valvula vaginalis, ce qui est visible d'après le mouvement conjoint de la paroi du vagin, la pointe est dirigée du côté dorsal et, au moyen d'un autre mouvement, on peut rabattre la valvule comme avec une sonde vaginale.

Pour aspirer le sperme, on peut faire pivoter la barre rectangulaire (L) longitudinalement et en même temps placer la seringue parallèlement à cette barre.

Adresse actuelle :

H. H.W. Velthuis. Laboratorium für vergleichende Physiologie, Jan van Galenstraat 40, Utrecht/Niederlande. 